\title{
Risk of elevated intraocular pressure and glaucoma in patients with uveitis; results of the Multicenter Uveitis Steroid Treatment Trial
}

\author{
David S. Friedman, MD, PhD $1,2,3$, Janet T. Holbrook, PhD, MPH${ }^{2}$, Husam Ansari, MD4, \\ Judith Alexander, BA ${ }^{2}$, Alyce Burke, MPH ${ }^{2}$, Susan B. Reed, BS ${ }^{5}$, Joanne Katz ${ }^{1,2,3}$, Jennifer \\ E. Thorne, MD, PhD ${ }^{1,2}$, Susan L. Lightman, PhD, FRCOphth ${ }^{6}$, John H. Kempen, MD, PhD ${ }^{4}$, \\ and for the MUST Research Group
}

${ }^{1}$ Dana Center for Preventive Ophthalmology, The Johns Hopkins University School of Medicine Wilmer Eye Institute ${ }^{2}$ Department of Epidemiology, The Johns Hopkins Bloomberg School of Public Health, Baltimore, Maryland ${ }^{3}$ Department of International Health, The Johns Hopkins Bloomberg School of Public Health, Baltimore, Maryland ${ }^{4}$ The Departments of Ophthalmology and Biostatistics \& Epidemiology, and the Center for Clinical Epidemiology and Biostatistics, The University of Pennsylvania Perelman School of Medicine, Philadelphia, Pennsylvania ${ }^{5}$ Fundus Photograph Reading Center, Department of Ophthalmology and Visual Sciences, University of Wisconsin, Madison, Wisconsin ${ }^{6} \mathrm{UCL} /$ Institute of Ophthalmology, and Hammersmith Hospital and the Royal Surrey County Hospital UK

\section{Abstract}

Objective-To report the two-year incidence of raised intraocular pressure (IOP) and glaucomatous optic nerve damage in patients with uveitis randomized to either fluocinolone acetonide (FA) implants or systemic therapy. Secondarily, to explore patient and eye characteristics associated with IOP elevation or nerve damage.

Design-A randomized, partially masked trial in which patients were randomized to either FA implants or systemic therapy.

Participants-Patients age 13 years or older with non-infectious intermediate, posterior or panuveitis active within the prior 60 days for which systemic corticosteroids were indicated were eligible.

(C) 2013 American Academy of Ophthalmology, Inc. Published by Elsevier Inc. All rights reserved.

Corresponding author: Janet T. Holbrook, PhD, MPH, The Johns Hopkins Center for Clinical Trials, 911 S Ann St., Baltimore, MD 2131, jholbroo@jhsph.edu.

*A full listing of the members of the MUST Research Group is available at http://aaojournal.org

Reprint Requests: Douglas A. Jabs, MD, MBA; MUST Chairman's Office; Department of Ophthalmology; Mount Sinai School of Medicine; One Gustave L. Levy Place, Box 1183; New York, NY 10029-6574; douglas.jabs@mssm.edu

Publisher's Disclaimer: This is a PDF file of an unedited manuscript that has been accepted for publication. As a service to our customers we are providing this early version of the manuscript. The manuscript will undergo copyediting, typesetting, and review of the resulting proof before it is published in its final citable form. Please note that during the production process errors may be discovered which could affect the content, and all legal disclaimers that apply to the journal pertain.

Conflicts of Interest: Dr. Friedman is a consultant to Bausch and Lomb, Dr. Kempen is a consultant for Alcon Laboratories, Allergan Pharmaceutical Corporation, Lux Biosciences, Inc., and Sanofi Pasteur SA, and XOMA Ltd. Dr. Thorne is a consultant for Allergan Pharmaceutical Corporation and XOMA Ltd. Professor Lightman is on the Boards and has received grants from Allergan

Pharmaceutical Corporation and GlaxoSmithKline plc. Drs. Holbrook and Katz and Ms. Burke, Alexander and Reed have no conflicts of interest.

This article contains online-only material. The following should appear online-only: Table 4, figures 4 and 5 and a listing of the members of the MUST Research Group. 
Methods-Visual fields were obtained at baseline and every 12 months using the Humphrey 24-2 SITA-fast protocol. Stereoscopic optic nerve photos were taken at baseline and at 3, 6, 12 and 24-month follow-up visits. IOP was measured by masked examiners at every study visit.

Main Outcome Measures-Glaucoma was diagnosed based on an increase in optic nerve cupto-disc ratio with visual field worsening or increased cup-to-disc ratio alone, when visual fields were not available for cases where visual field change was not evaluable, due to missing data or severe visual field loss at baseline.

Results-Most patients were treated as assigned, among those evaluated for glaucoma 97\% and $10 \%$ of patients assigned to implant and systemic treatment, respectively, received implants. More patients (65\%) assigned to implants experienced an IOP elevation of at least $10 \mathrm{mmHg}$ versus $24 \%$ assigned to systemic treatment $(\mathrm{P}<0.001)$. Similarly, $69 \%$ of patients assigned to the implant required IOP lowering therapy versus $26 \%$ in the systemic group $(\mathrm{P}<0.001)$. Glaucomatous optic nerve damage developed in $23 \%$ versus $6 \%(\mathrm{P}<0.001)$ of implant and systemic patients, respectively. In addition to treatment assignment, black race, use of IOP-lowering medications and uveitis activity at baseline were associated with incident glaucoma $(\mathrm{P}<0.05)$.

Conclusion-Implant-assigned eyes had about a four-fold risk of developing IOP elevation $\geq 10$ $\mathrm{mmHg}$ and of incident glaucomatous optic neuropathy over the first two years compared to those assigned to systemic therapy. Central visual acuity was unaffected. Aggressive IOP monitoring with early treatment (often including early filtration surgery) are needed to avoid glaucoma when vision-threatening inflammation requires implant therapy.

\section{Introduction}

Uveitis frequently results in elevated intraocular pressure (IOP) ${ }^{5,6}$, which has multiple etiologies but the majority is open angle presumed to be due to damage to trabecular meshwork function from the chronic inflammatory process. Furthermore, corticosteroid treatment, regardless of route, used to manage uveitis may raise IOP. 1,2,3,4 The Multicenter Uveitis Steroid Treatment (MUST) Trial compared the benefits and risks of fluocinolone acetonide intravitreous (FA) implants ( $0.59 \mathrm{mg}$, Bausch \& Lomb, Rochester, NY) to those associated with systemic treatment as recommended by guidelines ${ }^{7}$ in a randomized, controlled, comparative effectiveness trial of treatment for non-infectious intermediate, posterior, and panuveitis. ${ }^{8}$ The primary results of the trial showed similar visual acuity outcomes in the two groups after two years of follow-up but substantially more ocular complications, including glaucoma, in patients assigned to implants. ${ }^{9}$ Here, we report additional results about the IOP, glaucoma-related and vision outcomes from this study.

\section{Methods}

The MUST Trial was a randomized (allocation ratio 1:1), partially masked, parallel treatment comparative effectiveness trial (clinicaltrials.gov identifier NCT00132691). All patients provided written informed consent; all governing institutional review boards provided approvals, which were updated annually. Patients ages 13 years or older (only 5 patients were younger than 18) who had non-infectious intermediate, posterior or panuveitis in one or both eyes active within the prior 60 days for which systemic corticosteroids were indicated were eligible. Those who required systemic therapy for non-ocular indications, had uncontrolled diabetes mellitus, had an allergy to study medications, or had uncontrolled glaucoma or advanced glaucoma damage were excluded. Patients were enrolled at 23 centers, 21 in the United States and one each in the United Kingdom and Australia. Visits occurred at baseline, one month, three months, and then every three months for at least two years, with additional visits as needed for clinical care. 
Patients were randomized to FA implant or systemic therapy; randomization was stratified by center and site of inflammation (intermediate vs. posterior or panuveitis). Patients with bilateral uveitis assigned to the implant group were to receive implants in each eye for which it was indicated. Systemic therapy was guided by expert panel guidelines, ${ }^{7}$ typically starting with high dose prednisone ( $1 \mathrm{mg} / \mathrm{kg} / \mathrm{day}$ up to $60 \mathrm{mg} /$ day of prednisone) and then tapering to low doses of prednisone of $7.5 \mathrm{mg} /$ day or less; immunosuppressive drugs were used when indicated.

Study-certified visual acuity examiners measured best-corrected visual acuity as the number of letters read from standard logarithmic visual acuity charts ${ }^{10}$; change in visual acuity from baseline to two years was the primary outcome. Other important outcomes reported here include IOP, incidence of glaucoma, visual field sensitivity (the Humphrey mean deviation statistic), ${ }^{11}$ and quality of life measures.

Certified personnel measured IOP twice using Goldmann applanation tonometry. If the measures differed by more than $2 \mathrm{mmHg}$, a third measurement was performed. If Goldmann IOP could not be obtained then Tono-Pen (Mentor Ophthalmics, Norwell, MA) was used; Tono-Pen measurement were performed twice and, if the measures differed by 3 or more $\mathrm{mmHg}$, a third measurement was taken. The mean of the IOP measurements was used in the analysis. Other than visits at 1 and 3 months, when post-operative signs were expected to be visible, visual acuity and IOP examiners were masked.

Visual field assessments were obtained at baseline and every twelve months in a dark room with one eye patched with the proper refractive error trial lens using the Humphrey 24-2 SITA-fast protocol. The evaluation was repeated if the results were unreliable or abnormal at baseline, or if the visual field, as judged by the study ophthalmologist, had worsened from baseline at any follow-up measurement.

Stereoscopic optic nerve color photographs were taken using Reading Center-certified cameras and imaging procedures at baseline and at three, six, and twelve month follow-up visits and annually thereafter. Because patients with significant media opacities were eligible to enroll in the trial, there were relatively larger proportions of eyes with missing data on characteristics derived from evaluations of fundus photographs. ${ }^{12}$ For example, cupto-disc ratio (CDR) could not be determined at baseline from fundus photographs in $27 \%$ of eyes with uveitis and $10 \%$ did not have gradable OCT scans.

The National Eye Institute Visual function 25-item questionnaire was used to generate a single composite score that ranges from 0 to 100 with 100 being the maximum visual function; a 4 to 6 point difference has been considered a clinically meaningful one. ${ }^{13}$ The SF-36 responses are coded as scores for eight domains (physical functioning, bodily pain, general health, vitality, mental health, and physical, social and emotional role functioning) and summarized using physical and mental component summary scores (PCS and MCS, respectively). The scores are scaled to population norms, with a mean of 50 and a standard deviation of 10; a difference of 3 to 5 points has been considered clinically meaningful for the PCS scale. ${ }^{14,15}$

When possible, glaucoma was diagnosed from optic nerve photographs taken at 3,6 and 12 months on the basis of an increase in optic nerve CDR in the setting of documented visual field worsening. In addition, all visual fields were reviewed. In cases where probable glaucomatous worsening of the visual field was present, further assessment of the clinical course was undertaken. We also requested photographs from all visits when the visual field worsened dramatically. In cases where there was no ability to detect visual field change either due to missing visual field data or severe visual field loss at baseline, increased cupto-disc ratio alone was used to identify incident glaucoma. Because most patients had some 
visual field loss at baseline-presumably due to damage from uveitis itself and from chronic macular edema ${ }^{16}$ and its sequelae-detection of new visual field defects was difficult and glaucoma was only diagnosed if there was a clear optic nerve change based on photography. One hundred eyes with either poor quality images or no images at baseline were able to be evaluated by the glaucoma specialist at times using the blurry baseline images or subsequent images to identify incident glaucoma and at times relying on stable visual fields to confirm no incident glaucoma if images were missing. One glaucoma specialist (DSF) reviewed the optic nerve photos and all notes on persons with a change in CDR of 0.1 or more for small nerves (those that were $<2$ standard deviations in size from the mean of the baseline images) or 0.2 or more for normal or large nerves (based on masked Fundus Photograph Reading Center evaluations) and categorized the findings as definitely, probably, possibly, or not consistent with glaucoma damage. For this analysis, those graded as definite or probably were classified as glaucoma. A second glaucoma specialist (HA) reviewed those found to have definite or probable glaucoma and a $32 \%$ random sample of non-cases; disagreements were adjudicated by consensus.

The data presented here were collected on patients for up to two years after the randomization. Previous reports provide more details on the design and primary results of the trial. ${ }^{8,9}$

\section{Statistical analysis}

All available data on the outcome measures of interest (e.g., IOP elevation, glaucoma surgery or glaucomatous changes as described above) were included in the respective analyses. Because we were able to retrieve previously missing images or visual field data, this report includes evaluations of 13 additional eyes for glaucoma than were not included in the primary publication. ${ }^{9}$ Unless specified otherwise, data were analyzed by assigned treatment. Characteristics of eyes and patients at baseline by treatment assignment were analyzed by Wilcoxon or Chi-square tests and after 2 years of follow-up data were analyzed by glaucoma status using logistic or linear regression including adjustment for the baseline value for categorical and continuous outcomes, respectively. Associations of events (IOP elevation or glaucoma) in pairs of eyes with uveitis were evaluated with binomial regression models accounting for patient-level clustering.

Associations of baseline characteristics with elevation of IOP by $10 \mathrm{mmHg}$ were evaluated with proportional hazards regression. ${ }^{17}$ Race, gender and age were included in the multivariate models. Other variables selected for inclusion in the multivariate model were chosen using manual forward selection and had a P-value for inclusion of $<0.10$. Variables with missing values for more than $5 \%$ of eyes and variables that were closely associated with the ophthalmologist assessment of active disease were excluded from selection models.

For evaluating the risk of glaucomatous changes, associations were evaluated with Poisson regression. ${ }^{18}$ Poisson regression was used because assessments for uveitis were made at discrete time points, 1 and 2 years, and all but 1 case were identified at 2 years. Because the number of cases of glaucoma was relatively small (only sufficient to support a small number of variables in a multiple regression model), variables for inclusion in the multivariate model were selected based on inspection of bivariate associations, and variables for which more than 5\% of data were missing were excluded from multivariate models. All models that included data from each eye with uveitis were adjusted for the correlation between eyes from the same participant using Generalized Estimating Equations. ${ }^{19}$ Statistical analyses used SAS (SAS/STAT User's Guide, Version 9.1; SAS Inc, Cary NC) and Stata/1C 11.1 (StataCorp LP, College Station, TX). 


\section{Results}

\section{Characteristics of the study population}

A total of 129 persons (245 eyes with uveitis) were randomized to the FA implant, 122 (95\%) of whom received at least one implant, and 126 persons (234 eyes with uveitis) were randomized to standard systemic therapy, 121 (96\%) of whom received systemic treatment. Bilateral implants were implanted in $82(64 \%)$ patients assigned to the implant. Fourteen patients assigned to systemic therapy eventually received implants (11\%, 23 eyes), 9 of whom received implants in both eyes. Data on elevation of IOP were available for 249 patients (467 eyes with uveitis). Data were available for review of glaucoma status for 120 patients (219 eyes) and 114 patients (207 eyes) for patients assigned to implant and systemic therapy, respectively (Figure 1). There were no meaningful differences in the baseline characteristics of the subgroup of patients who were evaluated for glaucoma versus the entire group of patients.

The two treatment groups were similar with regard to age, sex, race, type of uveitis, and baseline ocular characteristics including visual acuity, CDR, and IOP. Eyes with uveitis in patients assigned to implants had borderline poorer visual field sensitivity than those in the systemic group (median -5.7 versus $-4.8 \mathrm{~dB}, \mathrm{P}=0.06$, respectively). Over $80 \%$ of eyes with uveitis had some diminution of visual field (mean deviation $<-3 \mathrm{~dB}$ ) at baseline. Conversely, visual acuity was good in most eyes and did not differ substantially between the two groups (Table 1).

Use of topical corticosteroid drops was common in both groups, $61 \%$ and $66 \%$ of patients assigned to the implant and systemic group, respectively, reported use of topical steroids at baseline. During follow-up 83\% and 79\% of patients in the implant and systemic group, respectively, used corticosteroid drops. Patients assigned to systemic therapy were more likely to receive periocular corticosteroids ( $48 \%$ versus $25 \%, \mathrm{P}<0.001)$ and intravitreal corticosteroids ( $18 \%$ versus $6 \%, \mathrm{P}=0.004)$ than patients assigned to implants.

\section{IOP elevation}

As reported previously, ${ }^{9}$ the incidence of IOP elevation and surgical treatment for elevated IOP was higher in patients (and eyes) assigned to the implant group (Table 2, Figure 2). Overall, $65 \%$ versus $24 \%$ of patients assigned to implant versus systemic therapy, respectively, experienced an elevation of at least $10 \mathrm{mmHg}$ above the baseline measurement within the first two years of follow-up. The mean peak IOP for those who experienced an increase in IOP of $10 \mathrm{mmHg}$ or more regardless of treatment assignment was $36 \mathrm{mmHg}$ (range $=14-70 \mathrm{mmHg}$ ). Over the 2 years, $69 \%$ of patients assigned to the implant received IOP lowering therapy as compared to $26 \%$ in the systemic treatment arm $(\mathrm{P}<0.001)$, and $32 \%$ versus $5 \%$ received a surgical intervention $(\mathrm{P}<0.001)$.

Nearly half of the patients assigned to implants (49\%) developed an IOP of $30 \mathrm{mmHg}$ or higher compared to $11 \%$ of patients assigned to the systemic group $(\mathrm{P}<0.001)$. Among patients who had a spike in IOP to $30 \mathrm{mmHg}$ or higher, similar percentages underwent surgery to lower IOP in the implant and systemic group (50\% vs $38 \%, \mathrm{P}=0.62$, respectively). Nineteen patients (15\%) assigned to the implant and $4(3 \%)$ assigned to systemic treatment had an IOP spike to $40 \mathrm{mmHg}$ or more; about half of those patients in both groups (53\%) subsequently had IOP-lowering surgery.

We restricted some analyses to patients who received their assigned treatment in order to investigate the timing of events relative to implant surgery. IOP elevations were common in the first year for patients assigned to and receiving implants (Figure 3); 58\% (70 patients) of those assigned to and receiving implants experienced an elevation of at least $10 \mathrm{mmHg}$ 
within 12 months of implant surgery as compared to $15 \%$ (17 patients) assigned to and receiving systemic treatment. Incident IOP elevation of $10 \mathrm{mmHg}$ or more occurred in 8 additional patients after 12 months in the implant group as compared to 7 additional patients in the systemic group. The median time from implant surgery to an IOP increase of at least $10 \mathrm{mmHg}$ was 9 months (95\% confidence interval (CI) 5 to12) in patients assigned to and receiving implants.

The risk of an IOP elevation or of having IOP lowering surgery was higher in the fellow eye of patients with bilateral implants once an event occurred in one eye. Among the 81 participants assigned to the implant group who received implants in both eyes, $22 \%$ had a 10 $\mathrm{mmHg}$ or greater increase in IOP in one eye while $49 \%$ developed an increase in both eyes. In participants with bilateral implants the risk for an increase in IOP in a fellow eye once an IOP elevation occurred in one eye was high (relative risk $(R R)=2.9,95 \%$ CI 1.7 to 4.9 , $\mathrm{P}<0.001)$. Ten patients $(12 \%)$ with bilateral implants underwent surgery for IOP lowering in one implanted eye and 20 patients (24\%) received IOP lowering surgery in both implanted eyes. The risk of having surgery was markedly increased for the fellow eye once one eye had surgery $(\mathrm{RR}=10.1,95 \% \mathrm{CI} 4.7$ to $21.8, \mathrm{P}<0.001)$.

\section{Risk factors for IOP elevation}

In bivariate analyses, factors associated with an increased risk of $10 \mathrm{mmHg}$ or greater elevation in IOP in eyes with uveitis were assignment to implant, age younger than 50 years, male gender, and baseline use of IOP-lowering drugs $(\mathrm{P}<0.05$, Table 3$)$. Phakic lens status and mean deviation of $-3 \mathrm{~dB}$ or worse were marginally $(0.05<\mathrm{P}<0.10)$ associated with IOP elevation risk (Table 4, available at http://aaojournal.org). Duration of disease, topical steroid use, bilateral disease, macular thickness and several other disease characteristics were not associated with altered risk of IOP elevation. Vogt-Koyanagi-Harada (VKH) disease was positively associated $(\mathrm{P}<0.001)$ while sarcoid-associated uveitis was negatively associated $(\mathrm{P}=0.04)$ (Table 4, available at http://aaojournal.org). In the multivariate model that included adjustments for treatment assignment, age, race, gender, receiving IOP medications at baseline and current inflammation activity, only implant assignment, younger age and baseline IOP medications were significantly associated; gender, mean deviation, and lens status were no longer associated (Table 3). Measures of vitreous cells, haze, and macular thickness were not included in multivariate exploratory analysis because of missing data.

\section{Incidence of glaucoma}

One glaucoma specialist reviewed all patient data (DSF) and a second (HA) reviewed all classified as having incident glaucoma as well as $119(32 \%)$ sets of photographs that had been diagnosed as "not glaucoma," by the initial reviewer. Only two $(1.7 \%)$ of these were re-categorized as glaucoma on re-review making it unlikely that many cases were missed during the initial assessment.

Glaucoma developed within 24 months in $16 \%$ vs. $4 \%$ ( $R R=4.5$, 95\% CI: 1.9-10.3) of eyes assigned to implant and systemic treatment, respectively (Table 2). With the exception of 4 eyes in 4 patients, all the cases of glaucoma observed were in eyes that received an implant (regardless of original treatment assignment). Twenty-seven (23\%) patients assigned to implant developed glaucoma, 9 in both eyes, compared to $7(6 \%)$ patients assigned to systemic treatment with only 1 case of bilateral glaucoma in that group. Among the eyes that developed glaucoma there were a few with dramatic worsening of the optic nerve damage over follow-up. Four eyes with a CDR of 0.5 or less at baseline developed a worsening in CDR of 0.4 or more. This occurred in 4 patients (out of 99 patients with CDR less than 0.5 
at baseline), all of whom had bilateral disease and all the affected eyes had been treated with an implant.

All cases of bilateral glaucoma were diagnosed in patients with bilateral implants. In the subset of patients with bilateral implants, the risk of glaucoma was increased in the fellow eye once glaucoma was identified in the first eye ( $R R=7.9,95 \%$ CI 3.4 to 18.9, $\mathrm{P}<0.001)$.

\section{Risk factors for glaucomatous optic nerve damage}

Baseline risk factors for glaucomatous optic nerve damage were assignment to implant, active uveitis, black race and IOP lowering medication use (Table $3, \mathrm{P}<0.05$ ). In addition, CDR greater than 0.5 and presence of anterior chamber cells of +0.5 or higher at baseline $e^{20}$ were marginally associated with increased glaucoma risk in bivariate models $(0.05<\mathrm{P}<0.10$, Table 4, available at http://aaojournal.org). Duration of disease, topical steroid use, bilateral disease, macular thickness and several other disease characteristics were not associated with development of glaucoma. In the multivariate model that included adjustments for treatment assignment, age, race, gender, use of IOP lowering medications and uveitis activity, only implant assignment, black race, use of IOP lowering medications and uveitis activity were significantly associated (Table 3 ). We did not include anterior cells in the multivariate model because it was strongly associated with uveitis activity and the presence of haze at baseline was not included because of missing data. Missing data (unable to see fundus) for the haze variable was associated with glaucoma diagnosis (Table 4, available at http:// aaojournal.org). As reported, elevated IOP during follow-up was common (Table 2) and it was strong risk factor for subsequent optic nerve damage; $24 \%$ of eyes with elevation of IOP of at least $10 \mathrm{~mm} \mathrm{Hg}$ during follow-up went on to develop glaucoma and $30 \%$ of eyes that developed an IOP elevation to $30 \mathrm{mmHg}$ or greater developed optic nerve damage.

Compared with eyes that did not develop glaucoma over two years of follow-up, eyes diagnosed with glaucoma had higher peak IOPs during follow-up as well as a higher IOP at the two year visit (Table 5, Figure 4 available at http://aaojournal.org). As expected since the diagnosis relied on CDR and, less so, on visual field, eyes diagnosed with glaucoma had greater impairment of visual field and increased CDR (Table 5). However, mean bestcorrect visual acuity in the eyes that developed glaucoma was not lower than those that did not (Table 5, Figure 5 available at http://aaojournal.org); $52 \%$ of eyes diagnosed with glaucoma and $60 \%$ of eyes without glaucoma had best-correct visual acuity of 20/40 or better at 2 years $(\mathrm{P}=0.36)$.

There were no differences in vision-related quality of life scores at two years between patients who developed glaucoma and those who did not (mean of 68 versus 70, respectively $\mathrm{P}=0.61$ ). Nor were there differences in the SF-36 physical component score (mean of 47 versus 47 , respectively $\mathrm{P}=0.92$ ) or the mental component score (mean of 51 versus 48 , respectively $\mathrm{P}=0.06$ ) between those who developed glaucoma and those who did not.

\section{Discussion}

FA implant therapy is associated with a high risk of substantial IOP elevation, often requiring glaucoma surgery. ${ }^{1}$ As in the prior drug licensing trials ${ }^{21,22,23}$, IOP elevation was common in eyes of MUST Trial participants receiving an implant, with nearly two thirds requiring IOP lowering therapy within two years and a quarter requiring surgery to control IOP. The estimated number needed to harm (NNH) was 5 (95\% 3 to 9), i.e., just about 1 of 5 patients treated with an implant rather than systemic treatment will have an IOP elevation of $10 \mathrm{mmHg}$ or higher that would not have occurred on systemic treatment. Our findings demonstrate that, within the first two years, these elevations can lead to glaucomatous optic nerve damage, which affected $23 \%$ of patients (16\% of eyes) assigned to 
receive an implant. The corresponding NNH for glaucoma was 8 (95\% CI 5 to 21), i.e., there will be 1 additional case of glaucoma for every 8 patients treated with an implant rather than systemic treatments. Within the 2 year period of follow-up, we did not see an effect of glaucoma on visual acuity, or vision-related or general health-related quality of life. The long-term prognosis of these individuals is uncertain and will require further study.

IOP elevation mostly occurred in the first year after implantation (median 9 months). However, new cases continued to occur in the second year, highlighting that the risk of IOP elevation is ongoing in these implanted eyes. Possible explanations for incident cases in the second year may be cumulative corticosteroid response, variability in the release of steroid from the implant over time and the natural history of treated uveitis. Extreme IOP elevations were seen in several instances, predominantly in patients assigned to the implant group. If left untreated, such elevations can result in rapid vision loss suggesting that patients with implants should be evaluated for IOP elevation frequently (the consensus of the MUST Research Group is that patients should be seen at least every six weeks and possibly more frequently). In patients with bilateral implants, the risk of a spike in IOP was markedly higher in the fellow eye after one eye had a spike; such patients would benefit from close observation and rapid intervention or in some cases, preemptive IOP lowering therapy.

Early elevations in IOP were expected since previous studies have documented that most cases occur in the first year, ${ }^{1}$ but recent publications indicate that sometimes extreme elevations can occur as late as the third year after implantation, ${ }^{24}$ consistent with our observations of high spikes in the second year after implantation.

Baseline factors associated with IOP elevation, after adjustment for confounding, included implant treatment assignment, younger age, and use of glaucoma medications at baseline. The association of younger age is consistent with the findings from an earlier study of intravitreal triamcinolone in persons with uveitis ${ }^{25}$ and clinical impression, but is difficult to explain. Perhaps younger eyes have greater aqueous secretion than older eyes, but an equal degree of corticosteroid-induced compromise of drainage, leading to greater likelihood of an IOP spike.

Our results confirm that the excess in IOP elevation seen in patients receiving implants does translate into a higher risk of glaucomatous optic neuropathy. The incidence of glaucoma in this study may not be generalizable to other settings where interventions may have been initiated earlier or later for elevations in IOP. Similar to the pattern observed with IOP elevation, the risk of glaucoma was markedly increased if the fellow eye was identified as having glaucoma. Patient's eyes were not evaluated independently, so the grading of the first eye may have influenced the evaluation of the second eye and hence the increase in risk noted in this study may be an overestimate of the risk in the fellow eye. Furthermore, the diagnosis was challenging in this patient population who frequently had visual field defects at baseline, and worsening of the visual field could have been due to progressive cataract, glaucoma or to the disease process itself. Nevertheless, the effect size associated with implant treatment, and second eye involvement in patients with one eye affected, is so large that it still is unlikely that these associations are spurious.

In addition to assignment to implant and IOP elevation during follow-up, other risk factors for glaucoma included black race, use of IOP-lowering medications at baseline, and active uveitis at baseline. Patients already using IOP-lowering medications may have had less outflow facility at the outset, and hence may have suffered earlier or more pronounced IOP elevation, with greater risk of glaucoma. A number of patients with active uveitis at baseline also may have had impaired aqueous secretion initially, which might have been restored with control of inflammation at about the same time aqueous outflow was impaired, leading 
to similar IOP effects and downstream effects on glaucoma risk. However, an evaluation of cases of uveitis likely to have ciliary body involvement did not show a consistent association (Table 4 available at http://aaojournal.org).

Furthermore, because of relatively high rates of missing data many anatomic characteristics of the eyes could not be included in multivariate models.

The high risk of IOP elevation and glaucoma should be considered when evaluating the appropriateness of FA implant for patients with uveitis, particularly those with any prior evidence of IOP control issues, glaucoma or large CDR. Patients on any IOP lowering medication at enrollment were at notably higher risk for optic nerve damage, and those on two or more medications were even more vulnerable. One prior report has suggested the approach of performing simultaneous filtering surgery in such patients, ${ }^{26}$ if fluocinolone acetonide implant therapy would otherwise be highly desirable. The MUST trial excluded persons with unmanageable IOP and those with advanced glaucomatous optic nerve injury, so our results likely underestimate the IOP and glaucoma risk following implant therapy in populations including such patients.

In conclusion, patients assigned to receive FA implant had 4 to 5-fold risk of developing large IOP elevations over the first two years compared to those assigned to receive systemic therapy and about one in six uveitic eyes in the implant group developed glaucomatous optic neuropathy in the context of at least quarterly monitoring in a clinical trial. The attributable risk of implant therapy on glaucoma was $12.6 \%$ in the MUST Trial, with one excess case of glaucoma for every eight patients assigned to implant therapy. Those who initially were on IOP lowering therapy were at higher risk as were those with active uveitis. Frequent monitoring for incident IOP elevation is indicated in patients receiving implant therapy, which potentially could mitigate much of this risk; the consensus of the MUST Research Group is that these patients should be followed at least every two months for IOP monitoring. Pre-emptive filtering surgery at the time of implantation ${ }^{26}$ should be considered in higher risk cases, and early use of filtration seems justified in patients experiencing substantial IOP elevations following implantation given that a large proportion will not be controllable with eye drops alone. These risks should be weighed against the benefits of implant therapy, which usually is successful in obtaining sustained control of inflammation in cases where other many options have failed, when determining the ideal therapy for patients with uveitis.

\section{Supplementary Material}

Refer to Web version on PubMed Central for supplementary material.

\section{Acknowledgments}

The authors wish to thank Lea Drye, PhD and Mark Van Natta, MHS for their assistance in the statistical analyses and Vinnette Morrison for her work on the evaluation of study images and visual field reports.

Financial Support: This study is supported by National Eye Institute Cooperative Agreements U10EY014655 (Dr. Jabs), U10EY014660 (Dr. Holbrook), and U10EY014656 (Dr. Altaweel). Bausch \& Lomb provided support to the study in the form of donation of fluocinolone implants for patients randomized to implant therapy who were uninsured or otherwise unable to pay for implants, or were located at a site where implants could not be readily purchased (e.g., in the United Kingdom). Additional support was provided by Research to Prevent Blindness (JET is the Sybil B. Harrington Special Scholar) and the Paul and Evanina Mackall Foundation. A representative of the National Eye Institute participated in the conduct of the study, including the study design and the collection, management, analysis, and interpretation of the data, as well as in the review and approval of this manuscript. 


\section{References}

1. Goldstein DA, Godfrey DG, Hall A, et al. Intraocular pressure in patients with uveitis treated with fluocinolone acetonide implants. Arch Ophthalmol. 2007; 125:1478-1485. [PubMed: 17923537]

2. Leder HA, Jabs DA, Galor A, et al. Periocular triamcinolone acetonide injections for cystoid macular edema complicating noninfectious uveitis. Am J Ophthalmol. 2011; 152:441-448. [PubMed: 21652023]

3. Ferrante P, Ramsey A, Bunce C, Lightman S. Clinical trial to compare efficacy and side-effects of injection of posterior sub-Tenon triamcinolone versus orbital floor methylprednisolone in the management of posterior uveitis. Clin Experiment Ophthalmol. 2004; 32:563-568. [PubMed: 15575824]

4. Lowder C, Belfort R Jr, Lightman S, et al. Ozurdex HURON Study Group. Dexamethasone intravitreal implant in the treatment of noninfectious intermediate or posterior uveitis. Arch Ophthalmol. 2011; 129:545-553. [PubMed: 21220619]

5. Sallam A, Sheth HG, Habot-Wilner Z, Lightman S. Outcome of raised intraocular pressure in uveitic eyes with and without a corticosteroid-induced hypertensive response. Am J Ophthalmol. 2009; 148:207-213. [PubMed: 19403113]

6. Herbert HM, Viswanathan A, Jackson H, Lightman SL. Risk factors for elevated intraocular pressure in uveitis. J Glaucoma. 2004; 13:96-99. [PubMed: 15097253]

7. Jabs DA, Rosenbaum JT, Foster CS, et al. Guidelines for the use of immunosuppressive drugs in patients with ocular inflammatory disorders: recommendations of an expert panel. Am J Ophthalmol. 2000; 130:492-513. [PubMed: 11024423]

8. Multicenter Uveitis Steroid Treatment Trial Research Group. The Multicenter Uveitis Steroid Treatment Trial: rationale, design and baseline characteristics. Am J Ophthalmol. 2010; 149:550 561. [PubMed: 20097325]

9. Kempen JH, Altaweel MM, Holbrook JT, et al. Multicenter Uveitis Steroid Treatment (MUST) Trial Research Group. Randomized comparison of systemic anti-inflammatory therapy versus fluocinolone acetonide implant for intermediate, posterior and panuveitis: the Multicenter Uveitis Treatment Trial. Ophthalmology. 2011; 118:1916-1926. [PubMed: 21840602]

10. Ferris FL III, Bailey I. Standardizing the measurement of visual acuity for clinical research studies: guidelines from the Eye Care Technology Forum. Ophthalmology. 1996; 103:181-182. [PubMed: 8628551]

11. Bengtsson B, Heijl A. Comparing significance and magnitude of glaucomatous visual field defects using the SITA and Full Threshold strategies. Acta Ophthalmol Scand. 1999; 77:143-146. [PubMed: 10321527]

12. Gangaputra SS, Altaweel MM, Peng Q, et al. MUST Trial Research Group. Morphologic assessment for glaucoma in the Multicenter Uveitis Steroid Treatment (MUST) Trial. Ocular Immunol Inflamm. 2011; 19:267-274.

13. Mangione CM, Lee PP, Gutierrez PR, et al. National Eye Institute Visual Function Questionnaire Field Test Investigators. Development of the 25-item National Eye Institute Visual Function Questionnaire. Arch Ophthalmol. 2001; 119:1050-1058. [PubMed: 11448327]

14. Brazier JE, Harper R, Jones NM, et al. Validating the SF-36 health survey questionnaire: new outcome measure for primary care. BMJ. 1992; 305:160-164. [PubMed: 1285753]

15. Jenkinson C, Wright L, Coulter A. Criterion validity and reliability of the SF-36 in a population sample. Qual Life Res. 1994; 3:7-12. [PubMed: 8142947]

16. Taylor SR, Lightman SL, Sugar EA, et al. The impact of macular edema on visual function in intermediate, posterior, and panuveitis. Ocul Immunol Inflamm. 2012; 20:171-181. [PubMed: 22530874]

17. Cox DR. Regression models and life-tables. J R Stat Soc Ser B. 1972; 34:187-220.

18. Cameron, AC.; Trivedi, PK. Regression Analysis of Count Data. Second Edition. Cambridge, UK: Cambridge University Press; 2013. p. 29-38.

19. Zeger SL, Liang KY. Longitudinal data analysis for discrete and continuous outcomes. Biometrics. 1986; 42:121-130. [PubMed: 3719049] 
20. Standardization of Uveitis Nomenclature (SUN) Working Group. Standardization of uveitis nomenclature for reporting clinical data. Results of the First International Workshop. Am J Ophthalmol. 2005; 140:509-516. [PubMed: 16196117]

21. Callanan DG, Jaffe GJ, Martin DF, et al. Treatment of posterior uveitis with a fluocinolone acetonide implant: three-year clinical trial results. Arch Ophthalmol. 2008; 126:1191-1201. [PubMed: 18779477]

22. Jaffe GJ, Ben-Nun J, Guo H, et al. Fluocinolone acetonide sustained drug delivery device to treat severe uveitis. Ophthalmology. 2000; 107:2024-2033. [PubMed: 11054326]

23. Jaffe GJ, Martin D, Callanan D, et al. Fluocinolone Acetonide Uveitis Study Group. Fluocinolone acetonide implant (Retisert) for noninfectious posterior uveitis: thirty-four-week results of a multicenter randomized clinical study. Ophthalmology. 2006; 113:1020-1027. [PubMed: 16690128]

24. Bollinger K, Kim J, Lowder CY, et al. Intraocular pressure outcomes of patients with fluocinolone acetonide intravitreal implant for noninfectious uveitis. Ophthalmology. 2011; 118:1927-1931. [PubMed: 21652079]

25. Kok H, Lau C, Maycock N, et al. Outcome of intravitreal triamcinolone in uveitis. Ophthalmology. 2005; 112:1916-1921. [PubMed: 16171868]

26. Malone PE, Herndon LW, Muir KW, Jaffe GJ. Combined fluocinolone acetonide intravitreal insertion and glaucoma drainage device placement for chronic uveitis and glaucoma. Am J Ophthalmol. 2010; 49:800-806. [PubMed: 20189158] 


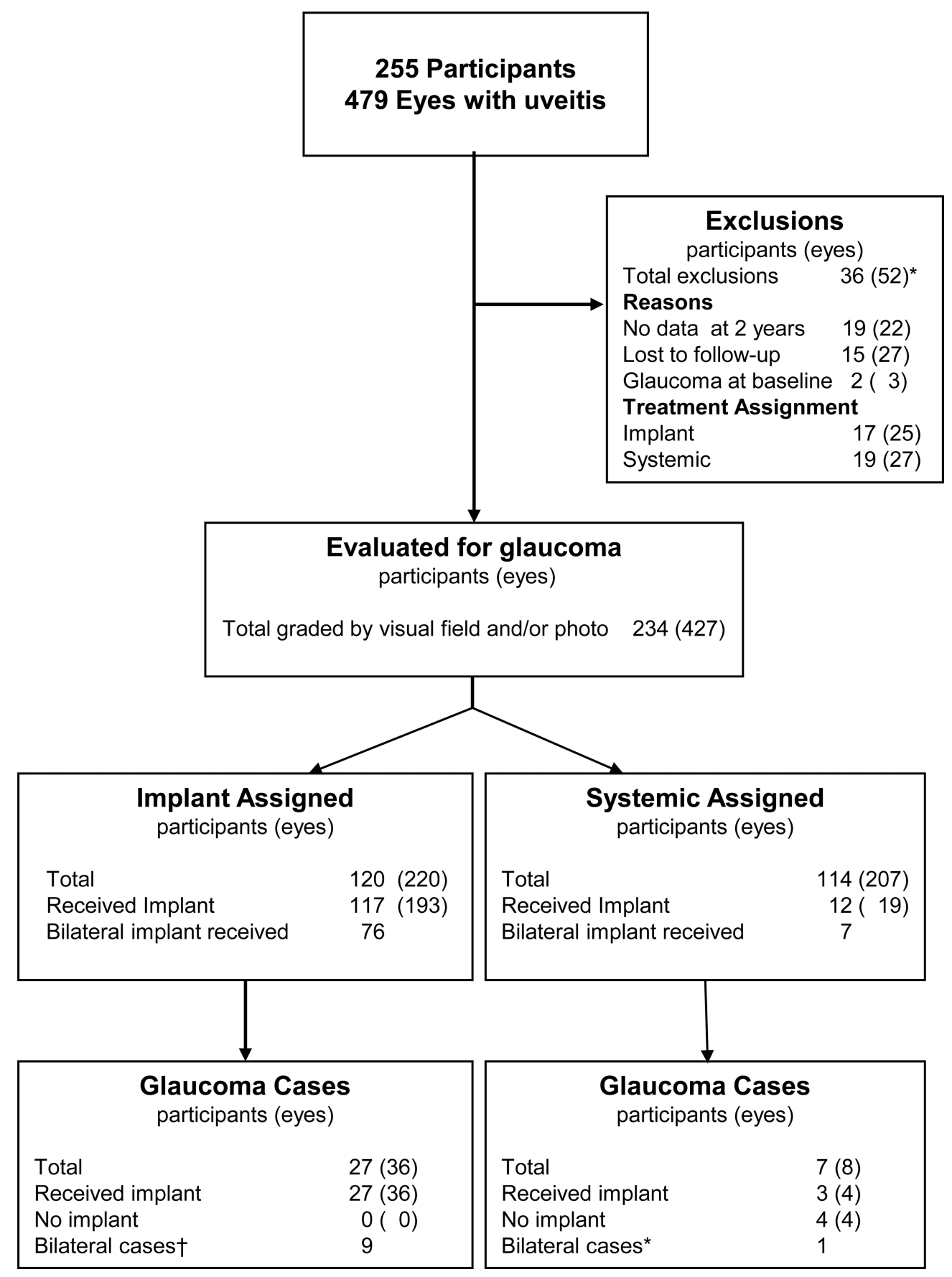

Figure 1.

CONSORT diagram; VF - visual field 


\section{Intraocular pressure (IOP)}

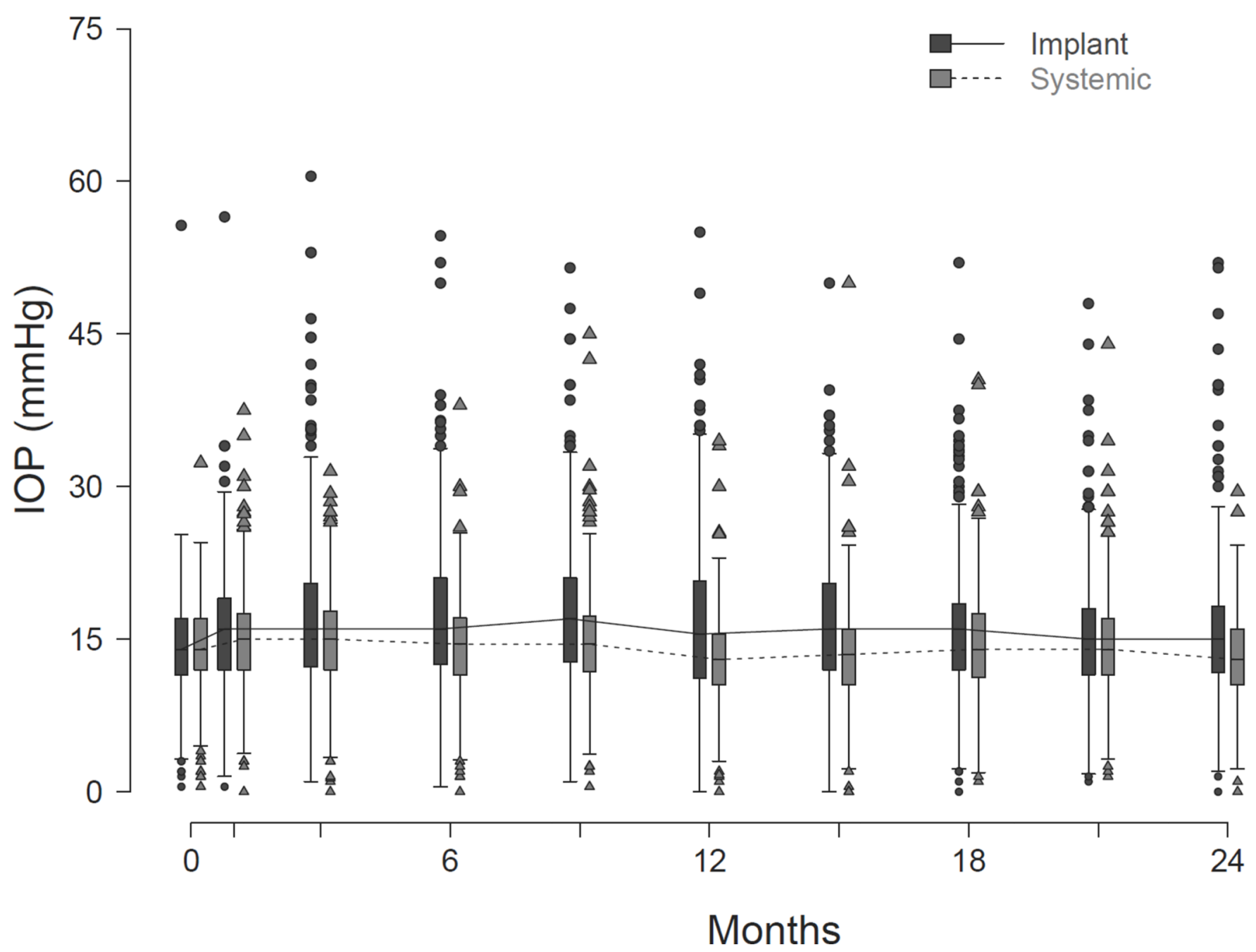

Figure 2.

Box plots of IOP measurements from eyes with uveitis during the trial by treatment assignment. Data for the Implant group are in black and data for the Systemic group are in grey. The middle bar of the boxes represents the median; the lower and upper ends of the boxes are the first and third quartiles, respectively. The whiskers represent values within 1.5 times the inter-quartile range from the upper or lower quartile (or the minimum and maximum if within 1.5 times the interquartile range of the quartiles) and data more extreme than the whiskers are plotted individually as outliers (circles for implant group and triangles for systemic group). 


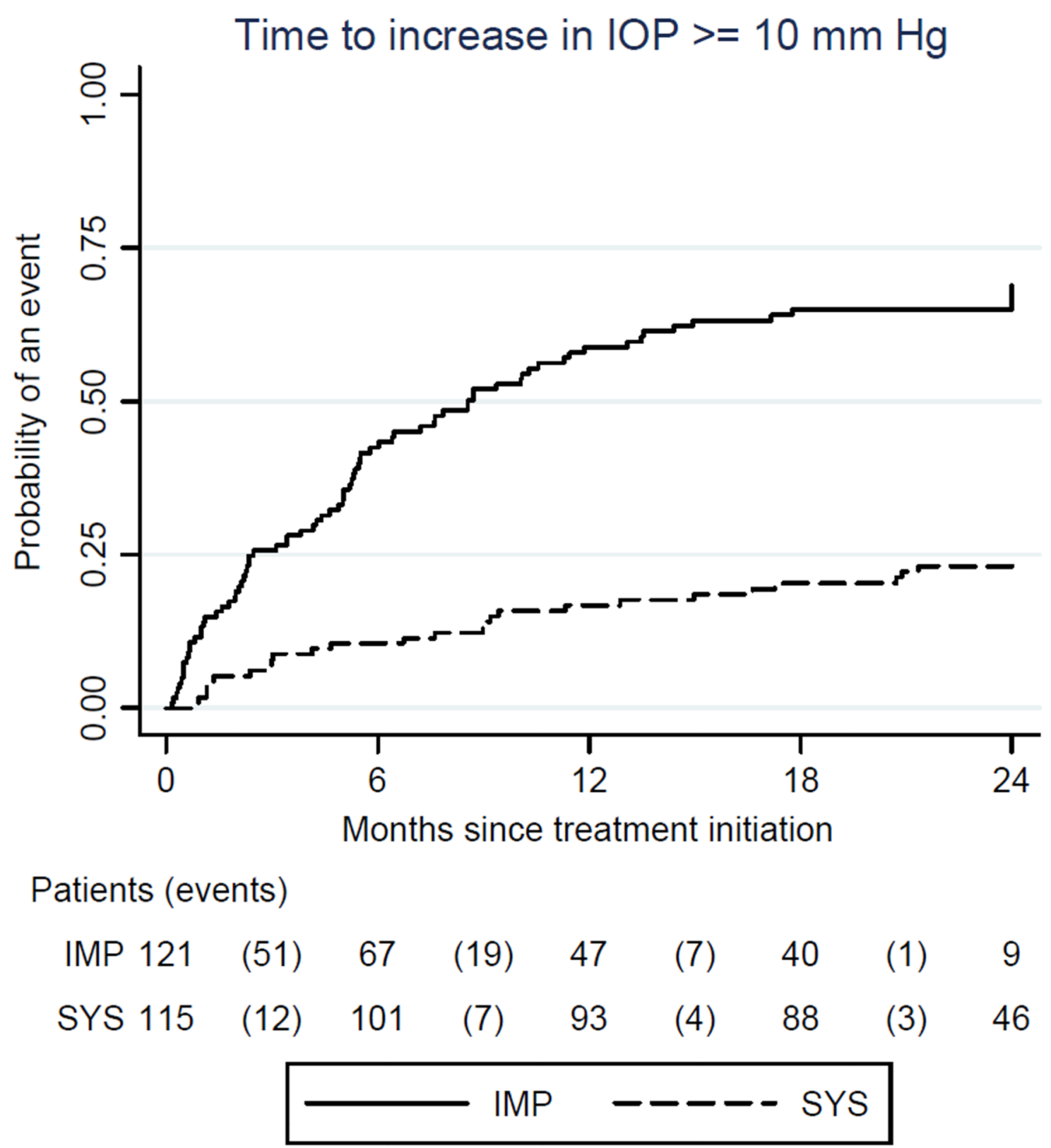

Figure 3.

Kaplan-Meir curve of time from treatment initiation to increase in IOP $\geq 10 \mathrm{~mm} \mathrm{Hg}$ by treatment assignment among patients who received their assigned treatment. The numbers below the graph represent the number of patients in the risk set at each time point and, in parenthesis, the number of events that occurred within the 3 month time intervals. Abbreviations: IOP - intraocular pressure, IMP - Implant treatment assignment, SYS Systemic treatment assignment 
Table 1

Baseline characteristics of participants and eyes with uveitis by assigned treatment

\begin{tabular}{|c|c|c|c|}
\hline Characteristic & Systemic & Implant & Total \\
\hline Demographics & $\mathrm{N}=126$ & $\mathrm{~N}=129$ & $\mathrm{~N}=255$ \\
\hline Age, mean years $(\mathrm{SD})$ & $46.9(15.1)$ & $45.8(15)$ & $46.3(15.0)$ \\
\hline Female Gender, N(\%) & $100(79 \%)$ & $91(71 \%)$ & $191(75 \%)$ \\
\hline \multicolumn{4}{|l|}{ Race/Ethnicity, N(\%) } \\
\hline White & $70(56 \%)$ & $72(56 \%)$ & $142(56 \%)$ \\
\hline Hispanic & $15(12 \%)$ & $18(14 \%)$ & $33(13 \%)$ \\
\hline Black & $31(25 \%)$ & $35(27 \%)$ & $66(26 \%)$ \\
\hline Other & $10(8 \%)$ & $4(3 \%)$ & $14(5 \%)$ \\
\hline VFQ-25, mean score (SD) & $62.6(20.7)$ & $58.4(21.4)$ & $60.5(21.1)$ \\
\hline \multicolumn{4}{|l|}{ Clinical Characteristics } \\
\hline Patient specific & $N=126$ & $N=129$ & $\mathrm{~N}=\mathbf{2 5 5}$ \\
\hline Unilateral uveitis, $\mathrm{N}(\%)$ & $17(13 \%)$ & $12(9 \%)$ & $29(11 \%)$ \\
\hline \multicolumn{4}{|l|}{ Site of uveitis, $N(\%)$} \\
\hline Intermediate & $47(37 \%)$ & $50(39 \%)$ & $97(38 \%)$ \\
\hline Posterior or Panuveitis & $79(63 \%)$ & $79(61 \%)$ & $158(63 \%)$ \\
\hline Systemic disease present, $\mathrm{N}(\%)$ & $33(26 \%)$ & $36(27 \%)$ & $69(27 \%)$ \\
\hline Using glaucoma medications, $\mathrm{N}(\%)$ & $17(13 \%)$ & $23(17 \%)$ & $40(16 \%)$ \\
\hline Eye specific * & $N=234$ & $N=245$ & $N=479$ \\
\hline $\begin{array}{l}\text { Visual acuity, standard letters, } \\
\text { median }\left(25^{\text {th }}, 75^{\text {th }} \text { percentile }\right)\end{array}$ & $71(53,81)$ & $68(46,79)$ & $70(49,80)$ \\
\hline $\begin{array}{l}\text { Visual field, mean deviation }(\mathrm{dB}) \\
\text { median }\left(25^{\text {th }}, 75^{\text {th }} \text { percentile }\right)^{\dagger}\end{array}$ & $-4.8(-8.7,-2.8)$ & $-5.7(-9.9,-3.2)$ & $-5.2(-9.6,-3.0)$ \\
\hline $\mathrm{IOP} \mathrm{mm} \mathrm{Hg}$, Mean $(\mathrm{SD})^{\dagger}$ & $14(4.5)$ & $14.1(4.8)$ & $14(4.7)$ \\
\hline Cup to disc ratio, Mean (SD $)^{\dagger}$ & $0.32(0.13)$ & $0.31(0.12)$ & $0.31(0.12)$ \\
\hline Aphakic/Pseudophakic, N(\%) & $105(45 \%)$ & $102(42 \%)$ & $207(43 \%)$ \\
\hline Ciliary body involved, $\mathrm{N}(\%)$ & $97(41 \%)$ & $114(47 \%)$ & $211(44 \%)$ \\
\hline Anterior chamber involved, $\mathrm{N}(\%)$ & $48(21 \%)$ & $48(20 \%)$ & $96(20 \%)$ \\
\hline History of hypotony, $\mathrm{N}(\%)$ & $12(5 \%)$ & $12(5 \%)$ & $24(5 \%)$ \\
\hline History of elevated IOP, $\mathrm{N}(\%)$ & $36(15 \%)$ & $33(13 \%)$ & $69(14 \%)$ \\
\hline On glaucoma medication, $\mathrm{N}(\%)$ & $28(12 \%)$ & $37(15 \%)$ & $65(14 \%)$ \\
\hline
\end{tabular}

*Ee specific characteristics are summarized for the 479 eyes with uveitis at enrollment.

${ }^{\dagger}$ Missing data: 12 (5\%) implant and 8 (3\%) systemic therapy eyes for mean deviation score; 2 implant eyes IOP; and 81(33\%) and 43 (18\%) systemic therapy eyes for cup to disc ratio

Abbreviations: IOP - intraocular pressure; SD - standard deviation; VFQ-25 - Visual Function Questionnaire, 25 items; mm Hg - millimeters of mercury; db - decibels 


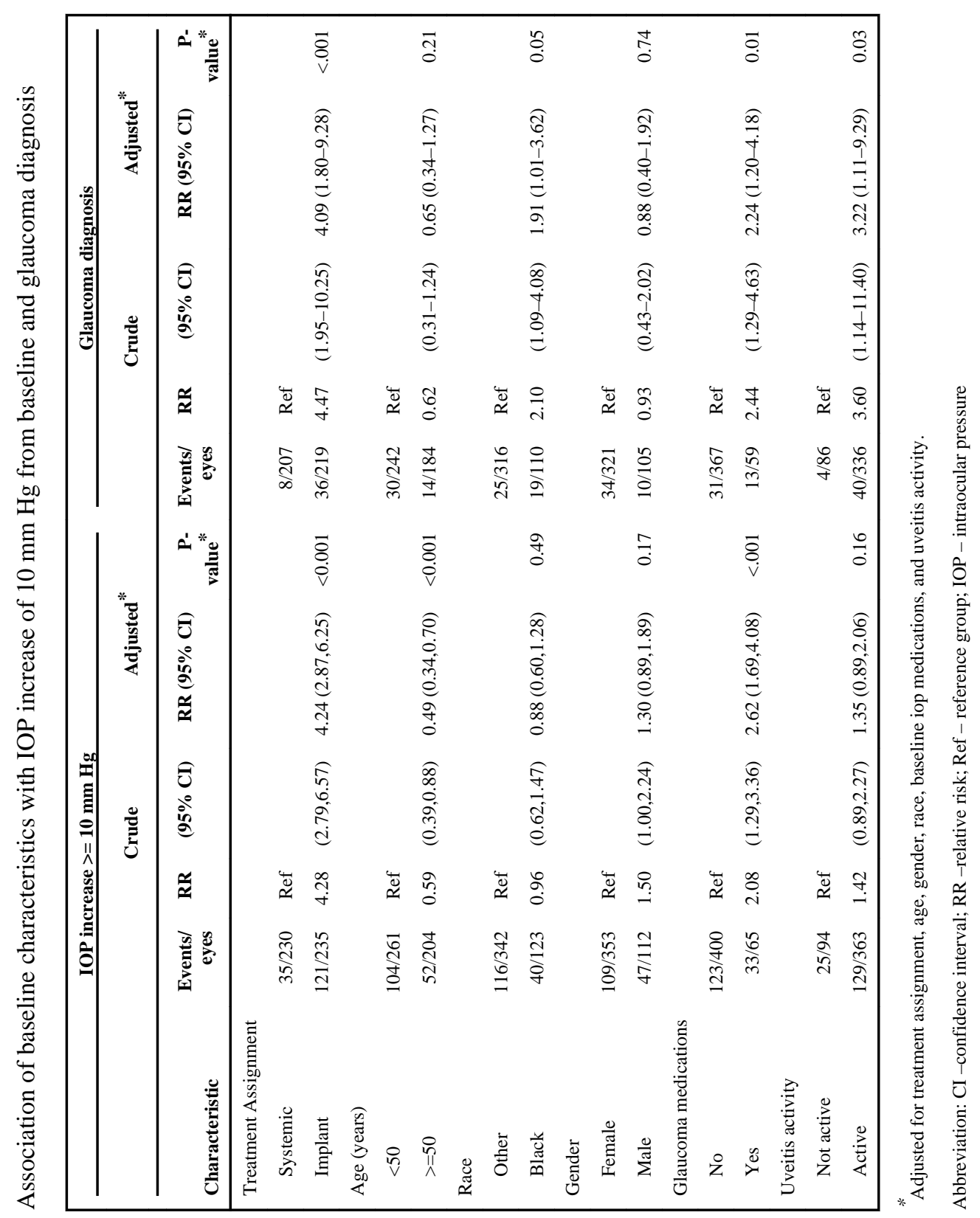


